\title{
Jardinería del Secreto. Esbozos para unos fundamentos bio-sociológicos del secreto
}

\author{
Gardening the Secret. Outline for Fundamentals \\ of a Bio-Sociology of Secrecy
}

\section{Francisco JaVier Gallego DueŃas}

Doctorando en el Departamento de Sociología I. UNED

mua2001es@yahoo.es (ESPAÑ)

Recibido: 01.03.2011

Aceptado: 28.12 .2011

\section{RESUMEN}

Utilizando el símil de la jardinería en la que la biología y la cultura se entretejen casi como en ninguna actividad humana, pretendemos avanzar algunas cuestiones en las que la perspectiva bio-sociológica puede hacer algunas aportaciones de interés al estudio del secreto. La filogénesis de los comportamientos implicados en el secreto, como la ocultación o el engaño puede ser clarificada desde la biología. La ontogénesis acabaría por señalar cómo, desde la bio-psicología, un humano concreto puede aprender a manejar secretos, así como los posibles beneficios bio-psico-sociales de guardar, compartir o romperlos, y el papel del secreto en la lucha por la vida. Quedaría el campo de investigación de posibles impulsos biológicos en el secreto y aquellas cuestiones biológicas sobre las que guardamos secretos los humanos.

\section{PALABRAS CLAVE}

Sociología del Secreto - Biosociología - Engaño — Filogénesis - Ontogénesis.

\section{ABSTRACT}

We have tried to propose several questions in which the bio-sociological perspective can make some interesting contributions on secrecy as same as gar- 
dening involves nature and culture. Biology can clarify concepts implied in behaviour phylogeny such as covering or deception. From a bio-psychological point of view, ontogeny would point out how a particular human being can learn secrets management, as well as the possible bio-psycho-social benefits of keeping, sharing or revealing them, and how secret shapes the struggle for life. There is still a lot to investigate on the issue of the possible biological drives over secrets and which biological matters human beings keep secrets about.

\section{KEY WORDS}

$$
\begin{aligned}
& \text { Sociology of Secrecy - Biosociology — Deception — Phylogeny — On- } \\
& \text { togeny. }
\end{aligned}
$$

Pretendemos, modestamente y de una manera breve, hacer recuento de aquellos puntos en los que confluyen, o deberían confluir, las ciencias sociales con la biología, especialmente desde la perspectiva evolucionista. Planteando cuestiones o temas que deben tratarse desde las ciencias sociales teniendo en cuenta ineludiblemente los desarrollos que se dan en el campo de la biología evolucionista.

La sociología del secreto fue fundada por Simmel $(1986,1908)$ ya hace cien años, resaltando las cualidades de nexo social que paradójicamente posee: ya que lo que separa, también une ${ }^{1}$.

Los estudios sobre el secreto abarcan diversos ámbitos, desde la política, (Shils, 1956), la literatura (Kermode, 1979), la jurisprudencia (Scheppele, 1988), la criptografía (Fabri, 2001), la antropología (Tefft, 1980), la historia (Vincent, 1998), la psicoterapia (Imber-Black, 1998), la psicología evolutiva (Van Mannen y Levering, 1999), y tratan también otros temas con los que el secreto se relaciona, como la vergüenza, la intimidad, la mentira...

Todas estas cuestiones (culturales) tienen un correlato biológico muy evidente. Como señalan Cosmides, Tooby y Barkow, «culture is not causeless and disembodied» (1995: 3). Aunque en relación a esto hemos ser muy cautos, puesto que la sociobiología tal como la instauró Edward O. Wilson ha recibido numerosas y fundadas críticas (Gould, 1978)². Por ello, quizás la concepción de la bio-sociología que propugna Anthony Walsh sea más pertinente:

\footnotetext{
${ }^{1}$ Es lo que Averill denomina pegamento social (Walsh, 1995; 70).

${ }^{2}$ Especialmente serias son aquellas que consideran que la descripción del mundo de las hormigas no es sino una antropomorfización. La evidencia de que hay insectos sociales de los que podemos deducir la biología de las sociedades humanas se convierte así en una tautología. El evolucionismo, al margen de los debates lamarckianos, comparte una característica con el funcionalismo en sociología. Ambos parten de que cada situación o acción tiene una función, ya sea evidente o latente. Pero eso es precisamente lo que se debería demostrar. La psicología evolucionista, por su parte, no sólo da por sentado que cada cosa tiene una función, sino que sabe cuál es esa función: servir al gen egoísta.
} 
«Biosociology is an emerging paradigm seeking to understand human behavior by integrating relevant insights from the natural sciences into tradicional sociological thinking. Biosociology is not a «biological» perspective; it is a biosocial perspectives that recognizes «the continuous, mutual, and inseparable interaction between biology and the social enviroment»» (Walsh, 1995: 1)

La mayoría de los historiadores y sociólogos son, en palabras de Shermen (1966), «cognitive creacionists», ya que creen que la mente (incluyendo la inteligencia, las emociones, las creencias y las actitudes) es inmune a las leyes de la naturaleza, ya sean biológicas o ambientales ${ }^{3}$. Lee Elis habla de biophobia (Freese, 2000: 6), mientras que Pierre L. van den Berghe se pregunta por qué la mayoría de los sociólogos no son ni quieren ser evolucionistas (Berghe, 1991: 259). Por su parte, Cosmides y Tooby hacen la crítica a lo que denominan el Modelo Estándar de las Ciencias Sociales, ejemplificado en Durkheim, para el que los hechos sociales sólo pueden ser explicados por hechos sociales, «omnis cultura ex cultura» (Lowie, citado en Cosmides y Tooby, 1995: 21) ${ }^{4}$.

Las relaciones entre Darwin y las ciencias sociales son tempranas. A pesar de que el concepto de supervivencia del más apto de Spencer es la base de la selección natural, se impuso hablar de «darwinismo social» (Castro, López-Fanjul y Toro, 2003) ${ }^{5}$. Aquí queremos manifestar la necesidad de ser, desde la sociología, «evolutionarly honest» (Freese, 2000: 436). Esto quiere decir que la teoria sociológica debe hacer el intento de asumir las bases biológicas y psicológicas de la evolución y no olvidar la naturaleza animal que nos conforma como seres sociales, sin que esto suponga un reduccionismo biológico (o psicológico) de ningún tipo.

\section{DEFINICIÓN DE SECRETO}

Provisionalmente vamos a definir el secreto distinguiendo entre el contenido y el acto de ocultar, para centrarnos en la acción de ocultar ${ }^{6}$. Un secreto es una práctica social en la que unos actores, en una determinada situación, evitan, limitan o modifican la comunicación de algo (una acción, un pensamiento, un sen-

\footnotetext{
${ }^{3}$ Citado en Freese (2000: 3).

4 Sin embargo, esa es la virtud que Gustavo Bueno define como «cierre categorial» para una ciencia (Quintanilla, 1976; 55).

5 Igual que el término «herencia», que proviene de lo cultural y luego pasó a la naturaleza, podríamos considerar el término «darwinismo social» (cuando lo correcto sería hablar de «spencerismo biológico») un concepto de ida y vuelta, tomando el símil del cante jondo. Junto a la evolución natural se habló de la evolución de la cultura (ej. Boas). Y aunque se prefiere la versión lamarckiana, en el caso de la evolución cultural podríamos aplicar el darwinismo básico: surgen multitud de ideas, teorías, ideologías, genéticamente distintas y sólo perviven las más aptas, las que sirven a varios amos. Freese propone que los principios de Darwin sean aceptados tanto en sus acepciones genéticas como metafóricas (2003: 18).

6 Asumiendo una «sociology of secrecy» más que una «sociology of secrets».
} 
timiento...) a otros actores durante cierto tiempo, haciendo uso de ciertas tácticas, es decir, suponiendo un esfuerzo. Dentro de estas tácticas encontramos el silencio, la mentira, el engaño, el ocultamiento...

En nuestra propuesta de definición evitamos usar el concepto de «conciencia», por que, a priori, no se descarta la posibilidad de encontrar usos del secreto en otras especies ( $\sin$ necesidad de que se tenga conciencia de estos) ${ }^{7}$. Sin embargo, recalcamos una condición inexcusable en el secreto: la información a la que se refiere este puede ser o no comunicada, es decir, para que exista un secreto debe darse esa posibilidad alternativa. En aquellos casos en los que algo no se transmite por incapacidad, por tratarse de un enigma irresoluble o por ser algo inconcebible, no estaríamos ante casos de secreto. Desechamos pues, por ejemplo, que el mimetismo del insecto palo sea una práctica de secreto en la medida en que no es intencional. Ahora bien, es necesario prestarle atención porque se puede considerar tanto un paso evolutivo previo, como un modelo para ocultamientos humanos (sociales).

Los biólogos están acostumbrados a regirse por la pauta de «los cuatro por qués» de Tinberger (Dunbar, 1998: 92), buscando las funciones y propósitos, los mecanismos, la ontogénesis y la filogénesis; así que nuestra propuesta deberá tener estos objetivos como horizonte.

En lugar de funciones, hablaremos de usos. Los secretos se usan de distintas maneras: se guardan, se comparten y se acaba con ellos; y cada uno de estos usos forma parte, a su vez, de un objetivo, de una estrategia. Se guarda un secreto para evitar un castigo, mantener el estatus o adquirir una ventaja... Se cuenta un secreto para buscar ayuda, para llevar a cabo una venganza... Tefft define el secreto como una estrategia para la adaptación conductual que implica mecanismos de copia que los humanos exhiben para obtener lo que quieren o ajustar sus vidas y propósitos (Tefft, 1980: 321).

\section{MECANISMOS, HERRAMIENTAS Y ARMAS}

El secreto, pues, utiliza una serie de herramientas: el silencio, el ocultamiento, la mentira (que no sólo es no decir la verdad, sino que incluye decir parte de la verdad o decirla de un modo que nadie la crea), o el engaño. Hay quienes prefieren, sin embargo, una definición restrictiva del «engaño» que incluya sólo procesos mentales, excluyendo adaptaciones biológicas o fenómenos socioculturales, para evitar problemas de explicaciones teleológicas.

Mitchell y Thompson (1986) recopilan en un volumen diferentes aportaciones de diversos autores al estudio de las conductas engañosas, que recoge ejemplos de avocetas, luciérnagas, crustáceos, macacos, pájaros tropicales, zorros ár-

${ }^{7}$ De esta forma ponemos el acento en la utilización de una táctica, que no tiene por qué ser consciente. Podemos encontrar múltiples ejemplos de usos no-conscientes de secreto: la base del psicoanálisis, por ejemplo, se encuentra en los secretos inconscientes.

EMPIRIA. Revista de Metodología de Ciencias Sociales. N. ${ }^{2}$ 3, enero-junio, 2012, pp. 117-136. ISSN: $1139-5737$ 
ticos, elefantes en cautividad, perros, chimpancés y otros primates, incluidos los humanos.

En dicho volumen, Chevalier-Skolnikoff (1986: 205) define el engaño (deception) como el envío intencionado de signos no verdaderos para obtener fines predeterminados a través de la conducta consecuente del receptor. En la teoría de sistemas, el engaño puede entenderse simplemente como el envío y/o recepción de información que induce a error ${ }^{8}$ (Anderson, 1986: 324). Anderson propone una distinción desde una perspectiva cibernética entre secreto y engaño, asumiendo que el secreto implica un ahorro de energía, y minimizando el hecho de que ocultar puede suponer también un gasto de energía ${ }^{9}$, mientras que en el engaño la información que se emite supone un gasto, temporal o parcial, hasta que el receptor pierde interés y la información engañosa se convierte en un lugar común que ya no es necesario ocultar (Anderson, 1986: 325).

Los psicólogos Dick Byrne y Andrew Whithen emplean el concepto «tactical deception» [engaño táctico] para dar cuenta de las ocasiones en las que un individuo intenta explotar a otro mediante la manipulación de su conocimiento de la situación (Byrne \& Whiten, 1988). Un caso de esto lo encontramos descrito por Sue Savage-Rumbaugh, en un estudio de larga duración de dos chimpancés, Austin y Sherman. Sherman intimidaba a Austin causándole gran angustia, hasta que un día Austin descubrió que Sherman se asustaba, especialmente por la noche, de los ruidos que provenían del exterior de sus habitaciones. A partir de entonces, cada vez que el acoso de Sherman se hacía insoportable, Austin salía corriendo y golpeaba vigorosamente desde fuera de su cubil y volvía apresuradamente lloriqueando y poniendo cara aterrorizada. Entonces Sherman, invariablemente, respondía con pánico y le pedía acurrucarse junto a él (Dunbar, 1997; 93).

Aunque Simmel consideraba el secreto como el rostro sociológico de la maldad moral, los aspectos éticos que implica su uso, desde el propio Simmel, son normalmente dejados a un lado, considerando sólo el secreto como forma, sub especie aeternitatis al margen de su contenido. El debate ético, en ningún modo, está zanjado en la biosociología (Hauser, 1999; Singer, 1981a, 1981b), siendo el atruismo el punto de atención más importante (Dawkins, 1993; Wilson, 1975, 2007; Castro y otros, 2008).

Deberíamos situar al secreto dentro de las conexiones biológico-evolutivas de las emociones morales, más allá de los inicios de Darwin, de Adam Smith y de los sueños de Dawkins. Concretamente, en la dinámica individuo/grupo, poniendo de relieve los riesgos de un uso egoísta del secreto así como los peligros del vínculo social ${ }^{10}$. Antony Walsh explica que la «comunidad» (Gemeinschaft)

\footnotetext{
${ }^{8}$ Mientras que el secreto parece estar más cerca de la no emisión de información. Sin embargo, esto debe matizarse. En realidad proponemos una versión inclusiva del concepto de secreto que pueda abarcar desde el silencio y el encubrimiento hasta la mentira.

9 Anderson admite a lo sumo un costo de «almacenamiento» («storage») o de «mantenimiento» (Anderson, 1986: 342).

${ }^{10}$ Pinker sostiene que las emociones que crean y salvan al grupo pueden ser tan terribles como
} 
presenta el sentido de pertenencia, la autoestima y un sistema de significados no problemáticos, pero descarta el logro de auto-realización mientras que la «asociación» (Gesselschaft), continúa Walsh, ofrece, por primera vez en la historia, la posibilidad, en el ámbito sociocultural, de un completo florecimiento de «la naturaleza humana» (Walsh, 1981; 261). Porque la motivación humana (sociocultural) está enraizada en la naturaleza biológica (en los genes y en la relación con el entorno) podemos hablar aquí de jardinería (actividad donde se entremezcla lo natural con lo socio-cultural).

\section{FILOGÉNESIS DEL SECRETO}

El nexo de unión entre los estudios biológicos y los sociales no se establece comprobando la existencia de secretos o conductas paralelas (a las sociales humanas) en comunidades animales, sino más bien determinando qué tácticas usadas por los humanos tienen un correlato biológico y planteando una integración vertical de las explicaciones, que abarque desde las más elementales formas de engaño hasta los más complejos secretos humanos (Cosmides, Tooby y Barkow, 1995: 5). Sobre esto, Frans de Waal explica que la diferencia de grado entre la inteligencia social alcanzada en el engaño de los monos frente al resto de los animales es enorme (la destreza del chimpancé es similar a la humana) mientras que los monos rhesus dan la impresión de ser casi trasparentes (de Waal, 1986: 240). Pero que pongamos de relieve una filogénesis no quiere decir, en absoluto, que no existan saltos cualitativos, aunque siempre dentro de una coherencia evolutiva.

Los etólogos (Mitchell, 1986: 3) han conseguido evidenciar conductas de engaño deliberado en una serie de niveles.

El nivel más bajo se da cuando un animal actúa porque no puede hacer otra cosa, cuando está «programado» para hacer algo. Así tenemos la cripsis o camuflaje, cuando la «apariencia» del animal se confunde con su entorno (como el insecto palo), y la mímesis, ya sea mimetismo batesiano (de serpientes que imitan los colores de la de coral) o mulleriano (a través del cual dos especies venenosas se asemejan y comparten señales de advertencia a los predadores). Mientras que la cripsis supone una adaptación evolutiva en la que sólo sobreviven los más desapercibidos; mediante el aposematismo (señales de advertencia), el animal sobrevive adoptando y exhibiendo rasgos formales que hacen desistir a los depredadores ${ }^{11}$. Sociológicamente podemos describir el uso del bisoñé como un

las que subrayan el individualismo, «cuando los dirigentes humanos han manipulado o coercionado a los individuos subsumiendo sus intereses a los del grupo, los resultados obtenidos son algunas de las peores atrocidades de la historia (Pinker, 2007: 521-522).

${ }^{11}$ Todos estos casos, según «el cuadrado de veridicción» de Greimas, que contrapone el secreto al engaño («l'illusoire») (Greimas y Courtes, 1979: 32), estarían más cerca del engaño que del secreto. Recordemos que en dicho cuadrado el secreto estaría en el ser y el no-parecer, mientras que el engaño estaría en el no ser y en el parecer. 
vestigio mimético que oculta secretamente los resultados del tratamiento de una enfermedad tabú.

El segundo nivel integra la percepción y las acciones que resultan del engaño. En este nivel, los organismos están programados para actualizar en ciertos momentos mecanismos de supervivencia, atacando aunque no puedan hacer daño, por ejemplo, o fingiendo estar heridos. Este tipo de conductas se llevan a cabo tanto en beneficio propio (por ejemplo, en algunos escarabajos rojos de la harina o en las zarigüeyas) como también en salvaguardia de las crías. A partir de este nivel sí se podrían rastrear actividades similares al secreto, ya que las actuaciones de los animales se corresponderían a prácticas en las que la información - en unos casos la situación de las crías y en otros su propia vida- es escamoteada al predador mediante una táctica que no se pone en marcha sino en el momento necesario. Las descripciones de Goffman (2003) de las estrategias de las personas estigmatizadas son claros ejemplos sociales para este segundo nivel.

El tercer nivel es un «programa abierto» que puede ser modificado por los resultados de las acciones del organismo, es decir, que muestra capacidad de aprendizaje, como esos pájaros que aprenden una gran variedad de cantos para dar la impresión de multitud y así evitar que se posen en su espacio otros pájaros. También tenemos el ejemplo del alcaudón, que emite falsas señales de peligro para alejar a competidores de la comida, o de la rana verde, que imita cantos más graves para parecer mayor. Entre los Estados podemos encontrar casos como los falsos tanques con los que Sadam engañó al ejército americano en la primera guerra del golfo.

El cuarto nivel de engaño incluye un programa abierto que es capaz de programarse y reprogramarse basándose en acciones pasadas o presentes del organismo: «intenta engañar» al «otro generalizado» (escondiendo la comida, por ejemplo). Este nivel es el prevalente en los humanos. Se da en los juegos de niños y en los de adultos, en el desarrollo del lenguaje y otras habilidades cognitivas, en las tácticas militares, las prácticas sexuales y las interacciones entre culturas y subculturas (Mitchell, 1986: 21-27).

William Searcy y Stephen Nowicki (2005) tratan diferentes aspectos del engaño, planteando la fiabilidad de los sistemas de señales que se dan entre los animales. Distinguen así entre aquellas señales que se dan cuando se solapan intereses (señales de alarma o llamadas de comida) y las que se dan cuando los intereses difieren (como los cantos de los pájaros) o incluso se oponen (como las falsas llamadas de las ranas). Plantean, por tanto, la cuestión de la honestidad de ciertas especies frente a otras. En conclusión, asumen que los animales pueden producir señales sólo si haciéndolo aumentan su propio bienestar, de igual manera que los que reciben esas señales responderán sólo si al hacerlo incrementan el suyo (Searcy y Nowicki, 2005: 207): es una cuestión, dicen, de maximización. Así, sólo saldrán triunfantes aquellas trasmisiones en las que los emisores consigan mayores recompensas que los receptores y eso sólo es posible identificando la fiabilidad de las señales. 


\section{ONTOGÉNESIS DEL SECRETO}

En el tratamiento del secreto desde la perspectiva del desarrollo individual, es decir, desde la perspectiva ontogenética, se suele hacer más hincapié en la utilidad y en los peligros de los secretos en las diferentes edades del individuo (como puede verse en la revisión bibliográfica de van Manen y Levering, 1999) que en describir el proceso de aprendizaje del secreto, descubriendo cómo y para qué se utiliza ${ }^{12}$. La biología debe explicar cuáles son los mecanismos por los que un individuo puede aprender el manejo del secreto, y debe articularse en esta tarea con la psicología evolutiva.

Teniendo en cuenta que la mentira es una de las estrategias utilizadas para guardar el secreto, podemos utilizarla como pista para elaborar un esquema del desarrollo evolutivo del secreto. No está claro cómo aprenden a mentir los niños, por lo que menos claro está aún cómo estos aprenden a manejar los secretos. Ajuriaguerra analiza la mentira en los niños dentro de los trastornos del comportamiento hacia los 6-7 años, «edad en la que se integran más sólidamente los valores sociales y morales» (Ajuriaguerra y Marcelli, 1992: 167). En el niño, sostenía Piaget, la distinción entre la verdad y la mentira es progresiva: antes de los 6 años, el niño no distingue entre mentira, actividad lúdica y fabulación. Lentamente, después de los 8 años, la mentira adquiere su dimensión intencional (Ajuriaguerra y Marcelli, 1992: 167-168). La mentira representa un verdadero aprendizaje progresivo en el que el lenguaje tiene un papel esencial: según Eco (2000: 22), un signo es cualquier cosa que sirve para mentir. TudorHart (1926) desarrolla una tipología de las mentiras: las «mentiras sociales», para beneficio de otros y de sí mismo; las «mentiras asociales», en beneficio de uno, pero sin perjudicar al otro; y las mentiras «antisociales», que dañan a otro (Vasek, 1986: 273).

Así, generalizando, podemos advertir que el niño pasa por diferentes etapas en el aprendizaje del secreto, aprendiendo a usarlo de diferentes maneras en diferentes contextos: la prohibición, la lealtad, la promesa.... El secreto puede aprenderse mediante juegos (cucú-tras, búsqueda del tesoro, escondite), por enseñanza directa («no se lo digas a mamá»), por condicionamiento («oculto algo porque cuando digo ciertas cosas me castigan»), por moldeamiento, mediante modelos, por imitación.... De la misma manera se enseña a reprimir la delación (Van Manen y Levering, 1999: 157). En el desarrollo de los comportamientos de competición/cooperación entre los niños, las redes se tejen con múltiples relaciones, coaliciones cambiantes y engaños (Flinn y Ward, 2005: 24). El advertir la conformidad o disconformidad de El Otro puede ser un instrumento de aprendizaje muy efectivo para el secreto.

Estas habilidades de aprendizaje pueden ser observadas, si bien en una forma

12 Esto es cierto excepto en el psicoanálisis, que se basa esencialmente en el secreto (Di Mascio, 1994; Nesse y Lloyd, 1995). Para Nesse y Lloyd, la represión hace decrecer la ansiedad, disminuyendo la conciencia de los deseos y hechos dolorosos (Nesse y Lloyd, 1995; 605).

EMPIRIA. Revista de Metodología de Ciencias Sociales. N. ${ }^{2}$ 3, enero-junio, 2012, pp. 117-136. ISSN: $1139-5737$ 
más simplificada, en los chimpancés. Lyn Miles, que estudia el uso del lenguaje en chimpancés, observa, en una serie de incidentes, posibles engaños de éstos. El esquema de análisis que propone Miles se compone de distintos niveles: el simple error, la sobre-extensión (casos similares, que sólo tienen la apariencia de un engaño), la asociación instrumental (una vez que el animal asocia un signo y un referente y comprueba que tras el signo obtiene una gratificación, puede hacer un uso bastardo del signo sin presencia del referente), el engaño intencional (aquí el chimpancé no sólo utiliza signos, sino que entiende que la interpretación del signo por parte del otro es diferente), y el engaño a través de falsas pistas (Miles, 1986: 262-263).

\section{CIRCULACIÓN DEL SECRETO: GUARDAR, CONTAR Y AVERIGUAR}

El secreto se puede guardar, compartir o contar. Foucault (1978: 74) no dudaba en calificar a la sociedad actual como una «sociedad singularmente confesante» (confesión y secreto, ya se sabe, van de la mano ${ }^{13}$ ). En nuestra investigación (en curso) sobre sociología del secreto hemos encontrado numerosas ocasiones en las que los sujetos ven como algo necesario el contar un secreto, dándose un impulso casi irrefrenable a compartirlo con alguien: por ello se valora positivamente la fortaleza de aquel que vence ese impulso, manteniendo el secreto ${ }^{14}$. Este impulso (social) a la confesión se puede rastrear en la biología: a él se refiere Theodor Reik (1959) cuando habla de una «compulsion to confess» en el plano estrictamente biológico.

Entendiendo la vida como una lucha por la supervivencia, el secreto forma parte indispensable de las estrategias de poder dentro de esta lucha. Como bien evidenció Foucault (1978), el poder se sirve del secreto para su ejercicio ${ }^{15}$, al tiempo que hace proliferar dispositivos de confesión. Desde una perspectiva bio-sociológica se debe también dar cuenta de cómo el poder es capaz de seducir, de incitar a la confesión, de crear la verdad sobre el sujeto.

En esta perspectiva, el estudio de «la curiosidad» ha sido objeto de importantes debates teóricos. El principal problema es conseguir una traducción operativa del concepto, por lo que se comenzó a hablar de «la conducta exploratoria». Inicialmente se debatió si esta se podía considerar un instinto o un impulso (Fowler, 1965: 20). Se realizaron experimentos en los que se comprobó que un aumento en la privación de alimentos (hambre) impulsaba de una manera notablemente alta las conductas exploratorias de los ratones en un laberinto (tipo $\mathrm{T}$ o

13 Para María Moliner en su diccionario la primera acepción de confesar era: «decir alguien una cosa que antes o a otras personas ha procurado ocultar».

14 Guardar un secreto era para Benjamin Franklin, según se cuenta, una de «las tres cosas más difíciles de esta vida.»

15 Desde los secretos de Estado, los secretos industriales y comerciales, el secreto de la burocracia y el saber médico, las sociedades secretas o la mística. 
Y), lo que llevó a distinguir entre dos tipos de exploración: la intrínseca y la extrínseca. En otros estudios se ponía el acento en la curiosidad como algo aprendido, y se la consideraba una reacción anticipatoria a los cambios en los estímulos contingentes de una acción instrumental (Fowler, 1965: 58). K. C. Montgomery propuso que la conducta exploratoria se desarrolla cuando un estímulo nuevo evoca en el organismo el impulso que la motiva. Este impulso puede disminuir con el tiempo y recuperarse tras un periodo sin estimulación (Montgomery, 1965: 104 y ss). Estos primeros estudios trataban de establecer la conducta exploratoria fuera de los rígidos parámetros del reflejo pavloviano.

La biología encuentra en los animales distintos casos de curiosidad ante lo secreto. Por ejemplo, Kaplan y Orians y Heerwagen explicitan, desde un punto de vista evolutivo, el impuso que genera el misterio en el paisaje, entendiéndolo como nuevo escenario. El «misterio» nos impulsa con la promesa de obtener una mayor información adentrándonos en lo desconocido (Kaplan, 1995: 588). Una característica de los paisajes es que incitan a buscar en su interior. Existe en ellos un lado oscuro que puede ser peligroso, pero que indudablemente es adaptativo, puesto que de esta forma se pueden conseguir mejores pastos o zonas de caza (Orians y Heerwagen, 1985: 573). Estos estudios establecerían la conexión biológica, quizás mínima, con las estrategias humanas de averiguar los secretos ajenos.

Por otra parte, se da también, Según Cosmides y Tooby (1995), una adaptación cognitiva para reconocer el engaño en los contratos sociales que protegió a nuestros ancestros de ser explotados en situaciones de intercambio social. Para demostrarlo proponen el «Wason Selection Test», mediante el que se comparan dos maneras de formular un mismo problema: una abstracta y otra «aplicada» en un contexto social ${ }^{16}$. Para Cosmides y Tooby, se activa un innato mecanismo de «look for cheaters» cuando nos encontramos en un contexto social, bajo un contrato social (Cosmides y Tooby, 1989: 84) ${ }^{17}$. Como los «tramposos» en situaciones de interacción pueden utilizar técnicas más o menos sofisticadas que incluyan secretos, este mecanismo nos remite al manejo del secreto en contextos sociales.

Los tramposos pueden fingir emociones, pero esto cuesta, como bien saben los actores que siguen el método Stanislavsky. Porque, como puntualiza Pinker, las emociones «probablemente se desarrollaron porque eran difíciles de fingir». Las emociones no son fácilmente controlables por razones evolutivas. Por ejemplo, si uno intentara reducir el ritmo cardíaco podría olvidarse de hacerlo la-

${ }^{16}$ Cuando a los sujetos se les inquiere para que apliquen una regla en su formulación abstracta (por ejemplo, «si en una cara de la carta aparece la letra D, en la otra debe haber un 3»), se producen multitud de errores de aplicación. En cambio, cuando esa regla tiene un enunciado contextualizado «socialmente» (por ejemplo, «si alguien bebe cerveza, debe tener más de 18 años»), apenas si se producen dudas (Cosmides y Tooby 1995: 181).

${ }_{17}$ Un contrato social, según Cosmides (1989: 197), expresa un intercambio en el que se requiere que un individuo pague un costo a otro individuo (o grupo) para que pueda recibir beneficios de ese individuo (o grupo).

EMPIRIA. Revista de Metodología de Ciencias Sociales. N. ${ }^{2}$ 3, enero-junio, 2012, pp. 117-136. ISSN: $1139-5737$ 
tir (Pinker, 2007: 533). Las emociones generan fatiga mental (ese es el principio del polígrafo). Por eso, remarcan tanto Pinker como Cosmides y Tooby, se recurre al autoengaño (selfdeception): «uno puede fugarse de sus intenciones ocultas si piensa que no son las suyas propias» (Pinker, 2007: 540). Como en el mecanismo freudiano de represión, las verdades que quieren permanecer en el secreto pasan al inconsciente ${ }^{18}$. La posibilidad de engaño que las palabras permiten al disociar actos y declaraciones se complementa y se incrementa con el autoengaño.

Una de las críticas que se le podrían hacer a la teoría del cheater-detector es que, de igual forma que estamos preparados genética y evolutivamente para advertir estos engaños, también lo estaríamos para mentir, ocultar o engañar (Cfr. Searcy y Nowicki, 2005). Ekman (1993) sostiene que no pueden existir rasgos físicos que delaten una mentira de forma tajante, puesto que de esa forma no podrían existir los mentirosos: siempre serían pillados. Como en las guerras de espías, a medida que se hacen más complejos los métodos de detección, más sofisticados se convierten los métodos de engaño (por ejemplo, Nesse y Lloyd, 1995: 606). Freese, por su parte, mantiene que no existe un «look for cheater» en nuestro cerebro (2000: 141 y ss.). Todo ello, según Pinker, forma parte de una «carrera armamentística» para regular el altruismo recíproco (Pinker, 2007: 520) y evitar así los usos antisociales del secreto.

Una explicación alternativa para dar cuenta del control de los tramposos, de los que usan artimañas de engaño en sus relaciones «sociales», igualmente incardinada en la perspectiva evolucionista, es la del antropólogo británico Robin Dunbar. El cerebro comparativamente mayor de los primates, dice Durban, tiene que ver con el manejo de las relaciones sociales. Por una parte, los grupos de conversación deben ser proporcionadamente más grandes que las camarillas convencionales de los primates que sólo se acicalan, como demuestran algunas investigaciones etológicas ${ }^{19}$. Por otra, se incrementa el tiempo de conversación que se dedica predominantemente al intercambio de información social: casi 2/3 de este tiempo se dedican a asuntos que tienen que ver con las relaciones sociales (gustos y fobias, experiencias personales, conductas de otra gente...) (Dunbar, 1997; 120). Este autor sugiere que el cuchicheo evolucionó como mecanismo de control de gorrones. Mediante el intercambio de información, los humanos son capaces de conseguir protección ante los aprovechados, llegando a avergonzarlos cuando su conducta deja de ajustarse a los estándares socialmente aceptados y es descubierta (Dunbar, 1997; 172). Dunbar apuesta en este descubrimiento por el lenguaje y la comunicación antes que por un innato mecanismo de detección.

Sin embargo, aunque desecháramos el detector de tramposos, eso no significa que no se puedan buscar raíces evolucionistas al secreto. Se debe hacer un

18 Se daría también esto ante la disonancia cognitiva (Festinger), que nos llevaría a ocultar lo que no nos cuadra, incluso en la construcción de nuestra propia imagen.

19 El acicalamiento es precursor del cuchicheo humano (Smith, 2007; 20). 
esfuerzo para hacer explícitas las premisas acerca de la motivación humana en las teorías sociológicas, y tratar, por ejemplo, el sentido de inclusión de grupo, la confianza, la autoconcepción o la gratificación simbólica o material (Turner, citado en Freese, 2000: 437). Existen, de hecho, estudios desde la sociobiología sobre la conducta antisocial y su predisposición genética al engaño y la mentira (Mealey, 1997), aunque en los manuales de psicología suelen evitar este tipo de consideraciones en la categorización de la personalidad. De cualquier modo, hay que tener en cuenta que una predisposición genética heredada siempre se ve activada por el aprendizaje del modelo de unos progenitores que también tienen esa predisposición, a la manera del agua que cae sobre unos surcos y los acentúa, por lo que aprendizaje y herencia se irían reforzando mutuamente. Y es en esos surcos donde surgen las especies que más tarde se irán modelando mediante la jardinería social.

\section{6. ¿BENEFICIOS? PSICO-BIO-SOCIOLÓGICOS DEL SECRETO}

Debemos advertir de la existencia de diferentes modelos a la hora de tratar el secreto desde el punto de vista de las relaciones sociales. Por un lado tenemos un modelo que ve necesario el secreto en la convivencia. Por otro lado existe una tendencia a considerar el secreto como nocivo, tendencia cuya aspiración máxima es la transparencia total en las relaciones: desde esta utópica perspectiva de transparencia comunicativa (Bretón, 2000) no se niega la existencia del secreto, pero se exige su fin.

Simmel marcaría una bisagra entre estas dos concepciones, al poner de relieve las cualidades integradoras de compartir un secreto, especialmente en las sociedades secretas, aunque también en la pareja o entre los amigos. El psicoanálisis sería el paradigma de esta opción: los secretos son inherentes al desarrollo humano (con la «escena primigenia» y el deseo edípico secreto), pero la salud desde el punto de vista biológico- consiste precisamente en acabar con ese secreto. La doctrina freudiana se basa en la idea de un intrínseco «malestar en la cultura», invirtiendo el dilema de Rousseau que sostenía que el hombre es bueno por naturaleza, pero que la sociedad lo corrompe. Pero ni Rousseau ni Freud son capaces de explicar por qué el hombre se encuentra a gusto entre sus congéneres.

En este punto, las explicaciones éticas y filosóficas deben, pues, dejar paso a la biología, pues será esta quien determine el «bienestar en la cultura», el bienestar que encuentran los individuos dentro de la cultura, pero no en cualquier cultura ni en cualquier situación, sino en el interior de burbujas atmopoiéticas (Sloterdijk, 2003), cuando entran en flujo (Castro Nogueira, 2008) con otros humanos que los seducen y los cobijan.

El aprendizaje assesor, por decirlo en términos castro-nogueirianos, va parejo al homo suadens. Los estudios biológicos sobre el cotilleo también remarcan la utilidad evolutiva que éste tiene. El cotilleo puede servir como mecanismo de control social, definiendo el grupo de pertenencia: «If no one tell you the gossip,

EMPIRIA. Revista de Metodología de Ciencias Sociales. N. o 23, enero-junio, 2012, pp. 117-136. ISSN: $1139-5737$ 
you are an outsider» (Barkow, 1995: 627). Aquí hay que advertir que el rumor (lo cotilleado) no siempre tiene como contenido un secreto (puede rumorearse una mentira, una información desconocida o incluso una conocida por todos), aunque en todo caso se comporta como si lo contuviese. Recordemos que sobre el secreto se puede actuar de varias formas, y compartirlo (es decir, contarlo o romperlo) es la más decisiva. Desde el punto de vista biológico evolutivo se pone de relieve la conexión entre compartir un secreto y el bienestar socio-biológico. El ser humano está programado genéticamente para encontrarse bien entre sus semejantes. En esta perspectiva encontramos el socius y el fluxus de Luis Castro Nogueira (2005: 547 y ss.) y la «animalidad propiamente humana» de la que habla José Luis Pardo (Pardo, 2004).

Desde el punto de vista biológico, el organismo considera el secreto como algo adaptativo simplemente considerando que disminuye la ansiedad ante el peligro. Ahora bien, el esfuerzo que supone mantener ese secreto también crea una ansiedad que puede ser disminuida al compartirlo. Compartir el secreto mantiene bajos los niveles de ansiedad ante el peligro, y disminuye también los creados por el esfuerzo de mantenerlos ${ }^{20}$. Las expresiones de «quitarse un peso» $\mathrm{o}$ «desahogarse», cuando se habla de compartir un secreto, explicitan este proceso biológico. Compartirlo (con otros, pero no con todos) sería un paso intermedio entre guardarlo - ya que en cierto círculo se seguiría manteniendo- y acabar con él — haciéndolo completamente público para que todo el mundo lo sepaAl compartir un secreto se recibe la recompensa de la empatía del grupo en el que este circula. Sin embargo, no debemos confundir el éxito biológico de conseguir ese decrecimiento del estrés, acompañado de un incremento paralelo de empatía, con el éxito evolutivo (Castro y otros, 2008: 186) ${ }^{21}$. Quedan por explicitar los beneficios evolutivos del sentimiento del secreto compartido, ya que «no todo nos da lo mismo» ${ }^{22}$ (Pardo, 2004: 45). Robin Dunbar $(1997,1998)$ explica cómo el contacto entre congéneres, mediante el acicalamiento mutuo o el chismorreo, fundamental en el desarrollo del cerebro, explicaría tanto el desarrollo del lenguaje como el de los mecanismos de control social: «chismorrear es uno

${ }^{20}$ La personalidad de los individuos más estresados, a los que afecta más al estrés, puede tener consecuencias sobre el mantenimiento del secreto: por un lado, estos serán más propensos a mantenerlo, para evitar la ansiedad de ser descubiertos; pero, igualmente, pueden ser buenos candidatos a contarlo, para evitar el estrés de mantenerlo. Este incontrolable efecto hace que el «secretismo» no sea tomado como un rasgo de personalidad en la mayoría de los test de personalidad conocidos.

21 Evolutivamente, el único amor importante es el amor por las crías (Thomas, 1985: 120). Walsh recoge los beneficios evolutivos del amor y otros sentimientos formando parte de estrategias $\mathrm{K}$ a la hora del cuidado de la prole, que encontramos en los chimpancés «casi con tanta extensión e intensidad como los humanos» (Walsh, 1995: 203). Fezter distingue un amor transparente por los hijos de un amor opaco por la pareja, en la que no es la persona en sí sino sus atributos los que despiertan ese sentimiento (Thomas, 1985: 117-118).

22 No tenemos más remedio que señalar como genéticas las diferencias en el gusto de cada humano, ya que no podemos atribuir a la cultura que a algunos les guste lo crudo y a otros lo cocido. 
de los pasatiempos favoritos en todas las sociedades humanas porque saber es poder» (Pinker, 2007: 690).

Anita Kelly recopila gran parte de la investigación realizada sobre psicología del secreto, y, en lugar de partir de la cuestión de por qué se producen los secretos, parte de la reflexión sobre por qué se revelan. Los motivos para contar los secretos incluyen la auto-clarificación ${ }^{23}$, la validación social, el desarrollo de las relaciones, el control social y la expresión —con el fin de conseguir la resolución del problema oculto-. El proceso de gastar energía para mantener información fuera del conocimiento de otra gente es lo que define el secreto, independientemente del tipo de secreto que se mantenga ${ }^{24}$. Aunque la habilidad para ocultar información puede verse como un signo de madurez o de funcionamiento normal de adulto, hay estudios que, entrando de lleno en lo biológico, relacionan la inhibición emocional con la secreción de cortisol, con problemas de corazón y con el funcionamiento del sistema inmunológico. Sobre estas cuestiones, utilizando la escala de Larson \& Chastain de «self-concealment $\rangle^{25}$, se demuestra que quienes alcanzan altas puntuaciones en esta escala tienen más problemas físicos y psicológicos que los que tienen puntuaciones más bajas. Finkenauer y Rime comprobaron que mantener un secreto predecía puntuaciones bajas en satisfacción vital (Kelly, 2002: 35). De forma paralela, Pennebaker y Susman descubrieron que los supervivientes a traumas infantiles que no hablaban de ellos, comparados con los que los comentaban, tendían a tener mayores problemas de hipertensión, cáncer y gripe. A corto plazo, por tanto, la represión, la contención de un secreto, puede tener beneficios, pero a la larga acarrea mayores problemas físicos, como dolores de estómago y náuseas.

Dentro de la psicología del secreto encontramos tres modelos básicos para dar cuenta de él y de sus efectos. El modelo de inhibición (Pennebaker) sostiene que inhibir activamente una conducta es estresante y se relaciona con la enfermedad. Así, cuando los individuos no pueden expresar sentimientos o pensamientos referidos a un evento traumático hay un incremento de pensamientos obsesivos acerca del evento, acompañado de un incremento de enfermedades. De manera opuesta, el acto de confianza que lleva a hablar del suceso, haciéndolo expresable, lo que reduce el estrés a corto plazo y a largo plazo reduce las enfermedades ${ }^{26}$. Por el contrario, en el modelo de preocupación (Lane y Wegner)

${ }^{23}$ Por «auto-clarificación» (self-clarification) nos referimos al deseo del que revela el secreto de re-conocer (o redefinir) su posición en el grupo.

24 Aunque es lógico pensar que mantener el secreto sobre un asesinato es presumiblemente más estresante que mantener un secreto sobre el consumo regular de marihuana (Kelly, 2002: 3 ).

25 De todos modos, hemos de decir, que dicha escala es francamente cuestionable.

${ }^{26}$ Pennebaker comprobó que contar los traumas, aunque sea por escrito, traía importantes beneficios para la salud, que dependían en parte de lo profundo de su expresión, ya que una cosa es no querer hablar de algo y otra no querer que otro lo sepa. Aunque no hay evidencia experimental de que mantener secretos cause problemas de salud, parece evidente que menteniendo un secreto una persona puede no obtener los beneficios de la salud que consigue al revelarlo. Parece, según ciertos estudios, que revelar un secreto con la explícita intención de ganar una nueva perspectiva ayuda a la gente a tener una sensación de resolución del problema que genera y contiene el secreto.

EMPIRIA. Revista de Metodología de Ciencias Sociales. N.o 23, enero-junio, 2012, pp. 117-136. ISSN: $1139-5737$ 
se constata que el hecho de intentar ocultar un pensamiento genera estrés y pensamientos que «sabotean» (thoughts intrusions) ese intento. Aunque Kelly comprobó que en ocasiones el ocultamiento puede ritualizarse y automatizarse sin apenas esfuerzo. En el modelo de auto-percepción el secreto se correlaciona con la vergüenza: el secreto conlleva una baja autopercepción de la valía personal del que lo guarda. Larson sugiere que la falta de apoyo social, junto con el ocultamiento, hace que el individuo mantenedor del secreto no se pueda recuperar del estrés que este genera. También encontramos en la psicología explicaciones que se basan en cierta predisposición a la ocultación y al aislamiento, haciendo incluso referencia a componentes genéticos. Kelly, por su parte, sugiere que es la clase de persona que mantiene secretos lo que hace a esta vulnerable a la enfermedad, en lugar de considerar que el hecho de guardar secretos sea lo que per se cause problemas de salud.

\section{SECRETOS BIOLÓGICOS}

Sólo si entretejemos la biología con la sociología podremos llegar a comprender los secretos relacionados con las adiciones, la reproducción artificial, las enfermedades y la muerte. No sólo el ambiente cultural y social determina la extensión del tabú sobre la enfermedad (de la enfermedad rodeada de secreto), estudiado, desde un punto de vista ensayístico, por Susan Sontag (2004). Los secretos ante la muerte o ante enfermedades limitantes y proscritas (incluso ante la incontinencia urinaria) serían imposibles de comprender sociológicamente si antes no se tiene conciencia del desarrollo biológico de estos trastornos. Desde la sociología, debemos a Goffman (2003) la descripción más completa del manejo del estigma como secreto.

Los «secretos de la vida y de la muerte», en expresión de Evelyn Fox Keller (1992), marcan también un cruce entre la biología y la sociología. Unos secretos que se conectan con el predomino masculino en la investigación tanto a nivel práctico (investigan varones) como teórico (denunciando el machismo de las expresiones y metáforas que pretenden desentrañar los secretos de la naturale$\mathrm{za})$.

Herdt (2003) describe los rituales secretos por los que el niño pasa a adulto. El secreto marca aquí la línea divisoria y es necesario para completar el desarrollo biológico de la «utopía masculina». Esta dimensión utópica se muestra en la Cabaña de los Hombres y la inseminación ritual. Gracias al secreto que la rodea, los varones pueden mantener su predominio en una sociedad cambiante: salvaguardan los mecanismos de reproducción de la masculinidad a la vez que ofrecen una imagen ideal de lo que la hombría significa.

La biología también se entreteje con la cultura en el ámbito de la tecnología reproductiva (fecundación artificial, donación de esperma y óvulos...) y en el de la adopción filial, en donde el secreto se maneja usualmente (Baran y Pannor, 1989).

EMPIRIA. Revista de Metodología de Ciencias Sociales. N. ${ }^{2}$ 3, enero-junio, 2012, pp. 117-136. ISSN: $1139-5737$ 


\section{CONCLUSIONES}

En los asuntos del secreto, sirviéndonos del símil de una labor de jardinería en la que la biología y la cultura se entretejen casi como en ninguna actividad humana ${ }^{27}$, debemos incorporar ambos aspectos con su voz propia, sin reduccionismos. Hemos pretendido avanzar algunas cuestiones en las que la perspectiva biosociológica puede hacer aportaciones de interés al estudio del secreto. La filogénesis de los comportamientos implicados en el secreto, como la ocultación o el engaño, puede ser clarificada desde la biología. La ontogénesis acabaría por señalar cómo, desde la bio-psicología, un humano concreto puede aprender a manejar secretos, descubriendo los beneficios bio-psico-sociales de guardarlos, compartirlos o desvelarlos, y el papel del secreto en la lucha por la vida. Quedaría abierto así un campo de investigación sobre posibles impulsos biológicos hacia el secreto y sobre aquellas cuestiones biológicas de las que guardamos secretos los humanos. Lo que de ningún modo se inscribe en nuestro horizonte es reducir a la biología los aspectos eminentemente sociales del secreto. Defendemos el secreto como una práctica social que, al igual que la práctica de la jardinería hace con plantas y parterres, aprovecha las cualidades biológicas del objeto tratado para un diseño plenamente humano y social, intencional si se quiere, llegando incluso a violentar las tendencias genéticas a través de una serie de variados diseños que pueden servir para ocultar, para recrearse o para mostrar la magnificencia del Poder Absoluto en Versalles.

\section{REFERENCIAS}

Ajuriaguerra, J. De y Marcelli, D. (1992): Psicopatología del niño. Barcelona. Masson.

Anderson, M. (1986): «Cultural Concatenation of Deceit and Secrecy» en Mitchell, Robert W. y Thompson, Nicholas (ed.): Deception perspectives on human and nonhuman deceit. Albany. State University of New York. Págs. 323-348.

BAran, A. y PAnnor, R. (1989): Lethal Secrets. The Shocking Consequences and Unsolved Problems of Artificial Insemination. New York. Warner Bros.

BARKow, J. H. (1995): «Beneath New Culture Is Old Psychology: Gossip and Social Stratification» en Barkow, Jerome H., Cosmides, Leda y Tooby, John: The Adapted Mind. Evolutionary Psychology and the Generation of Culture. New York-Oxford. Oxford University Press. Págs. 627-637

Barkow, J. H., Cosmides, L. y Tooby, J. (1995, 1992): The Adapted Mind. Evolutionary Psychology and the Generation of Culture. New York-Oxford. Oxford University Press.

Berghe, P. L. VAn Den (1991): «Sociology» en Maxwell, Mary (ed.) : The Sociobiological Imagination. New York. State University of Newy York Press. Págs. 269-282

27 Utilizamos la metáfora del «gardening» propuesta por Gross (2001) para entender la sociología de Simmel.

EMPIRIA. Revista de Metodología de Ciencias Sociales. N. ${ }^{2}$ 23, enero-junio, 2012, pp. 117-136. ISSN: $1139-5737$ 
Byrne, R. W. y Whiten, A. (eds.) (1988): Machiavellian Inteligence. Oxford. Oxford University Press.

Breton, P. (2000, 1992-1997): La utopía de la comunicación. Buenos Aires. Nueva Visión.

CAstro, L., López-Fanulu, C. y Toro, M. Á. (2003): A la sombra de Darwin. Madrid. Siglo XXI.

Castro Nogueira, L., Castro Nogueira, L. y Castro Nogueira, M. Á. (2008): ¿Quién teme a la naturaleza humana?. Madrid. Tecnos

Castro Nogueira, L., Castro Nogueira, M. Á. y Morales Navarro, J. (2005): Metodología de las ciencias sociales. Una introducción crítica. Madrid. Tecnos.

Chevalier-Skolnikoff, S. (1986): «An Exploration of the Ontogeny of Deception in Human Beings and Nonhuman Primates» en Mitchell, Robert W. y Thompson, Nicholas (ed.): Deception perspectives on human and nonhuman deceit. Albany. State University of New York. Págs. 205-220.

Cosmides, L. (1989): «The Logic of Social Exchange. Has Natural Selection Shaped How Human Reason?: Studies with the Wason Selection Task» en Cognition (31): Págs. 187-276

Cosmides, L.; ToOby, J. y BArkow, J. H. (1995): «Introduction: Evolutionary Psychology and Conceptual Integration» en Barkow, Jerome H.

Cosmides, L. y Tooby, J.: The Adapted Mind. Evolutionary Psychology and the Generation of Culture. New York-Oxford. Oxford University Press. Págs. 3-15

Cosmides, L. y Tooby, J. (1995): «Cognitive Adaptations for Social Exchange» en Barkow, Jerome H., Cosmides, Leda y Tooby, John: The Adapted Mind. Evolutionary Psychology and the Generation of Culture. New York-Oxford. Oxford University Press. Págs.163-228.

Dawkins, R. (1993, 1976): El gen egoísta. Barcelona. Salvat.

Di Mascio, P. (1994): Le maître de secret. Essai sur l'imaginaire théorique de Freud. L'Or d'Atlante. Champ Vallon.

Dunbar, R. (1997): Grooming, Gossip, and the Evolution of Language. Cambrige, Massachusetts. Harvard University Press.

DunBAR, R. (1998): «Theory of Mind and the Evolution of Language» en Hurford, J., Studdert-Kennedy, M., and Knight, C. (eds.): Approaches to the Evolution of Language: Social and Cognitive Bases. Cambridge University Press. Págs. 92-110.

Eco, U. (2000): Tratado de semiótica general. Barcelona, Lumen. $5^{\text {a }}$ edición.

Ellis, B. J. y GJoRKLund, D. F. (eds.) (2005): Origins of the Social Mind. Evolutionary Psychology and Child Development. New York, Guilford Press.

Ekman, P. y Frank MARK, G. (1993): «Lies That Fail» en Lewis, Michael y SAARnI, Carolyn (eds.): Lying and Deception in Everyday Life. New York. The Guilford Press. Págs.184-200.

FlinN, M. V. y WARD, C. V. (2005): «Ontogeny and Evolution of the Social Child» en Ellis, Bruce J. y Gjorklund, David F. (eds.): Origins of the Social Mind. Evolutionary Psychology and Child Development. New York, Guilford Press.

Foucault, M. (1978): Historia de la sexualidad. 1. La voluntad de saber. Madrid. Siglo XXI.

Fowler, H. (1965): Curiosity and Exploratory Behavior. New York-London. The Macmillan Company, Collier-Macmillan.

Freese, J. (2000): What Should Sociology Do About Darwin? Evaluating Some Potential Contributions of Sociobiology and Evolutionary Psychology to Sociology. Summit- 
ted to the faculty of the University Graduate School in partial fulfillment of the requirements for the degree Doctor of Philosopy. Indiana University.

Goffman, E. (2003, 1963): Estigma. La identidad deteriorada. Buenos Aires. Amorrortu

Goud, S. J. (1978): «Sociobiology: the art of storytelling» en New Scientist, 16 November.

Greimas, A. J. y Courtés, J. (1979): Semiótica. Diccionario razonado de la teoría del lenguaje. Madrid. Gredos.

Gross, M. (2001): «Unexpected Interactions Georg Simmel and the Observation of Nature» Journal of Classical Sociology (November 2001 vol. $1 n^{o}$. 3). Págs. 395-414.

Hauser, M. D. (2001): Wild Minds. New York. Henry Hold and. Company.

HERDT, G. H. (2003): Secrecy and Cultural Reallity: Utopian Ideologies of the New Guinea Men's House. Michigan. University of Michigan.

IMBER-BLACK, E. (1998): The secret life of families: truth-telling, privacy, and reconciliation in a tell-all society. New York, Bantan Books.

Inglis, D., Bone, J. y WiLKIE, R. (ed.) (2005): Nature. Critical Concepts in the Social Sciences. Vol IV. Reconfiguring the Social/Natural Interface. New York. Routledge.

KaPlan, S. (1995): «Environmental Preference in a Knowledge-Seeking, KnowledgeUsing Organism» en Barkow, Jerome H., Cosmides, Leda y Tooby, John: The Adapted Mind. Evolutionary Psychology and the Generation of Culture. New York-Oxford. Oxford University Press. Págs. 581-598.

KelLER, E. F. (1992): Secrets of Life, Secrets of Death. New York-London. Routledge.

Kelly, A. E. (2002): The Psychology of Secrets. New York. Kluwer Academic/Plenum Publishers

KERMODE, F. (1979): The Genesis of Secrecy On the Interpretation of narrative. London. Harvard University Press

MaXwell, M. (ed.) (1991): The Sociobiological Imagination. New York. State University of Newy York Press.

Miles, H. L. (1986): «How Can I Tell a Lie? Apes, Language, and the Problem of Deception» en Mitchell, Robert W. y Thompson, Nicholas (ed.): Deception perspectives on human and nonhuman deceit. Albany. State University of New York, P. 245266.

Mitchell, R. W. (1986): «A Framework for Discussing Deception» en Mitchell, Robert W. y Thompson, Nicholas (ed.): Deception perspectives on human and nonhuman deceit. Albany. State University of New York. Págs. 3-40.

Mitchell, R. W. y Thompson, N. (ed.) (1986): Deception perspectives on human and nonhuman deceit. Albany. State University of New York.

MEALEY, Linda (1997): «The sociobiology of sociopathy: an integrated evolutionary model» en Baron-Cohen, Simon (ed.): The Maladapted Mind: Classic Readings in Evolutionary Psychopathology. Págs. 133-187.

MONTGOMERY, K. C. (1965): «Exploratory Behavior as a Function of «Similarity» of Stimulus Situations» reproducido en Fowler, Harry: Curiosity and Exploratory Behavior. New York-London. The Macmillan Company, Collier-Macmillan. Págs. 104128.

Nesse, R. M. y Lloyd, A. T. (1995): «The Evolution of Psychodynamic Mechanisms» en Barkow, Jerome H., Cosmides, Leda y Tooby, John: The Adapted Mind. Evolutionary Psychology and the Generation of Culture. New York-Oxford. Oxford University Press. Págs. 601-624.

Orians, G. H. y HeERwagen, J. H. (1985): «Evolved Responses to Landscapes» en 
Barkow, Jerome H., Cosmides, Leda y Tooby, John: The Adapted Mind. Evolutionary Psychology and the Generation of Culture. New York-Oxford. Oxford University Press. Págs. 554-579.

PARDO, J. L. (2004): La intimidad. Valencia. Pre-textos.

PInKeR, S. (2007, 1997): Cómo funciona la mente. Barcelona. Destino.

Quintanilla, M. A. (dir.) (1976): Diccionario de filosofía contemporánea. Salamanca. Ediciones Sígueme.

ReIK, T. (1959): The Compulsion to Confess. New York. Farrar, Straus and Cudahy.

RusE, M. (1985): Sociobiology and Epistemology. Netherlands. Kluwer.

SEARCy, W. A., y Nowicki, S. (2005): The Evolution of Animal Communication: Reliability and Deception in Signaling Systems. Princeton. Princeton University Press.

SHILs, E. A. (1956): The Torment of Secrecy. London. William Heiman.

Simmel, G. (1986, 1908): Sociología. Estudio sobre las formas de socialización. Madrid. Alianza. 2 vols.

Singer, P. (1981a): «Ethics and Sociobiology» en Philosophy and Public Affairs. II, n ${ }^{\circ} 1$, Págs. 40-64.

Singer, P. (1981b): «The Biological Basis of Ethics» en Singer, Peter: The Expanding Circle: Ethics and Sociobiology. Oxford. Oxford University Press. Chapter 2.

SloterdiJK, P. (2003, 1998): Esferas I. Burbujas. Madrid. Siruela.

Smith, V. (2007): Clean. A History of Personal Hygiene and Purity. Oxford. Oxford University Press.

Sontag, S. (2004): Ante el dolor de los demás. Madrid. Punto de Lectura.

Spradlin, W. W. y Portenfield, P. B. (1979): Human biosociology. From Cell to Culture. New York-Heidelberg-Berlin. Springer-Verlag.

Tefft, S. K. (ed.) (1980): Secrecy. A Cross-Cultural Perspective. New York. Human Sciences Press

Thomas, L. (1985): «Love and Morality: The Possibility of Altruism» en Ruse, Michael (1985): Sociobiology and Epistemology. Netherlands. Kluwer. Págs. 115-129

Tooby, J. y Cosmides, L. (1995): «The Psichological Foundations of Culture» en Barkow, Jerome H., Cosmides, Leda y Tooby, John: The Adapted Mind. Evolutionary Psychology and the Generation of Culture. New York-Oxford. Oxford University Press. Pág. 19-136.

Tudor-Hart, B. E. (1926): «Are there cases in which lies are necessary?» Pedagogical Seminary, 33, 586-641.

TURNER, J. H. (2005): «Classical sociology and the restoration of nature: the relevance of Émile Durkheim and Georg Simmel» en Inglis, David, Bone, John y Wilkie, Rhoda (ed.): Nature. Critical Concepts in the Social Sciences. Vol IV. Reconfiguring the Social/Natural Interface. New York. Routledge.

VAN MANen MAX y LeVering, BAS (1999): Los secretos de la infancia. Intimidad, privacidad e identidad. Barcelona. Paidós.

VINCENT, D. (1998): The culture of secrecy. Britain, (1832-1998). Oxford. Oxford University Press.

WALSH, A. (1981): Human Nature and Love. Biological, Intraphychic and Social-Behavioral Perspectives. Washington. University Press of America.

WALSH, A. (1995): Biosociology: an Emerging Paradigm. Westport, Connecticut-London. Praeger.

WeGner, D. M. y LANE, J. D. (1995): «From secrecy to psychopathology» en J. W. Pen- 
nebaker (Ed.), Emotion, disclosure, and health Washington, DC: American Psychological Association. pp. 25-46.

WILSON, E. O. (1975): «Some Central Problems of Sociobiology» en Social Science Information, 1975, enero; vol. 14. Págs. 5-18 versión online disponible en http://ssi.sagepub.com/.

WILSON, E. O. (2007): «Rethinking the Theoretical Foundation of Sociobiology» en The Quarterly Review of Biology, december 2007, vol. 82, nº 4. Págs. 327-348. 